\title{
Adaptive Resolution Simulation in Equilibrium and Beyond
}

\author{
Han Wang \\ CAEP Software Center for High Performance Numerical Simulation, Beijing, China and \\ Zuse Institute Berlin, Germany
}

\author{
Animesh Agarwal \\ Institute for Mathematics, Freie Universität Berlin, Germany
}

\begin{abstract}
In this paper, we investigate the equilibrium statistical properties of both the force and potential interpolations of adaptive resolution simulation (AdResS) under the theoretical framework of grand-canonical like AdResS (GC-AdResS). The thermodynamic relations between the higher and lower resolutions are derived by considering the absence of fundamental conservation laws in mechanics for both branches of AdResS. In order to investigate the applicability of AdResS method in studying the properties beyond the equilibrium, we demonstrate the accuracy of AdResS in computing the dynamical properties in two numerical examples: The velocity auto-correlation of pure water and the conformational relaxation of alanine dipeptide dissolved in water. Theoretical and technical open questions of the AdResS method are discussed in the end of the paper.
\end{abstract}

* wang_han@iapcm.ac.cn 


\section{INTRODUCTION}

Adaptive resolution simulation (AdResS) [1-9] is a concurrent multiscale simulation method for molecular system. The terminology "concurrent multiscale simulation" means that part of the system is simulated with higher resolution molecular models for the purpose of accuracy, and in the meanwhile the rest of the system is simulated with lower resolution models for the purpose of saving computational cost. AdResS is, therefore, suitable for the systems where different physical phenomena is happening concurrently at different time and length scales. For example, the structure of a protein and its solvation shell should be resolved at the atomistic level, while far away form the protein, only the hydrodynamic properties of the solvent are of interest, which can be studied with satisfactory accuracy with very coarse-grained or even continuum models [10-12]. Similar techniques sharing the concurrent idea of multiscale simulation can be found, for example in Ref. [13 17].

The time evolution of a classical molecular systems is modeled with Newtonian dynamics, and fundamental conservation laws are satisfied, i.e. the momentum and energy conservation. However, in the AdResS system, due to the spacial change of molecular resolution, the two conservation laws cannot be satisfied in the same system [18, 19]. Traditionally, the AdResS scheme follows the force interpolation approaches that preserves the momentum conservation while breaks the energy conservation. Recently, alternative approaches based on potential interpolation, which preserve energy conservation while break the momentum conservation, were designed [20, 21]. The choice between the two AdResS branches therefore depends on the application. For example, in the cases of studying hydrodynamics, the momentum conservation is preferred to the energy conservation. However, if only the equilibrium properties are of interest, the question should be asked on the ensemble that AdResS samples, rather than directly on the mechanical conservation laws. In this context, it is worth noting that energy conservation is not a must for theoretical analyses on the equilibrium statistical properties, although out of the energy conservation one can define an auxiliary Hamiltonian [20, 22] (not a physical one [23]), which reduces the mathematical difficulties. In Sec. III the force and potential interpolations are investigated under the same theoretical framework GC-AdResS (GC stands for "Grand-Canonical like") [20] that raises necessary conditions for a grand-canonical sampling with AdResS scheme. In Sec. IV the equilibrium thermodynamic relations between the resolutions are provided for both branches 
of the AdResS. From these relations, it is noticed that the absence of mechanical conservation laws modifies the way of thermodynamic equilibrium between the resolutions.

The GC-AdResS framework answers how to accurately compute the equilibrium properties. However, although the system is in equilibrium, some properties, which are called dynamical properties, cannot be simply computed from the equilibrium ensemble averages. A typical example is the velocity auto-correlation. Moreover, the time-scales of conformational transformations of bio-macromolecules (e.g. protein and DNA) are, again, not equilibrium averages. The investigation of these dynamical properties are out of the scope of the GC-AdResS framework, and to the best of our knowledge, there is no theoretical answers on how accurately AdResS reproduces the dynamical properties of a system. Therefore, it is of practical significance to provide numerical evidence on the performance of AdResS in computing the dynamical properties. In Sec. V] we investigate the velocity auto-correlation in an AdResS water system, and the leading relaxation time-scales and corresponding conformational dynamics of a model dipeptide system. The importance of designing the thermostat in these simulations is also discussed. The paper is closed by a concluding section that mainly discusses the open problems in AdResS from both theoretical and practical perspectives.

\section{THE HYBRID RESOLUTION: BRIDGING THE MOLECULAR MODELS OF DIFFERENT RESOLUTIONS}

In most cases discussed in this work, we refer to higher resolution as the classical atomistic model, while the lower resolution as the coarse-grained molecular model. However, one should keep in mind that the "atomistic" and "coarse-grained" resolutions may refer to more general molecular models, e.g. the higher resolution can be path-integral representation [5], and lower resolution can be continuous hydrodynamic description [12].

In AdResS scheme, the simulation region $\Omega$ is decomposed without overlapping into three regions: the atomistic region $\Omega_{\mathrm{AT}}$, the coarse-grained region $\Omega_{\mathrm{CG}}$, and a hybrid region $\Omega_{\mathrm{HY}}$ bridging the former two regions. That means the atomistic region is always connected to the coarse-grained region via a hybrid region. The names of the atomistic and coarse-grained regions are self-explanatory, while in the hybrid region each molecule has both the atomistic and coarse-grained resolutions. A schematic plot of an AdResS simulation is presented in Fig. 1. The AdResS uses a scalar weighting function $w(\boldsymbol{r}), \boldsymbol{r} \in \Omega$ to denote the resolution 


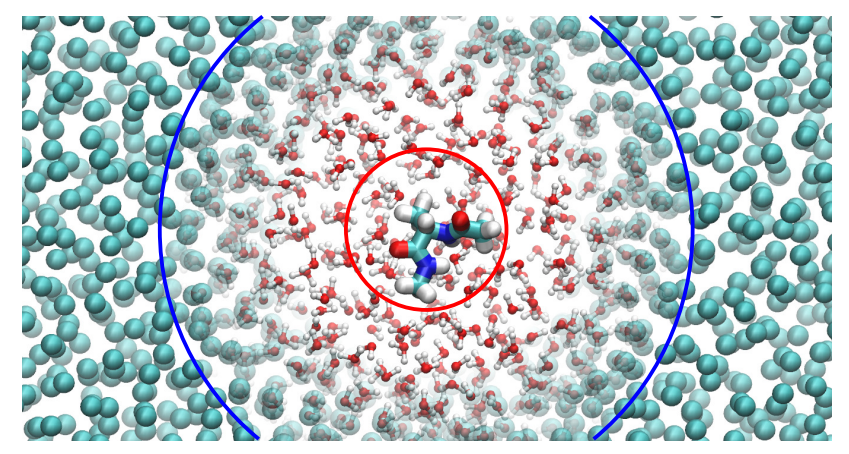

FIG. 1. A schematic plot of an AdResS simulation. In side the red circle is the atomistic region. Between the red and blue circles is the hybrid region. Outside the blue circle is the coarse-grained region.

of the molecule at position $\boldsymbol{r}$. The definition of the weighting function is not unique, one possible and perhaps the most popular choice is

$$
w(\boldsymbol{r})= \begin{cases}1 & \boldsymbol{r} \in \Omega_{\mathrm{AT}} \\ \cos ^{2}\left[\frac{\pi}{2} \cdot \frac{\operatorname{dist}\left(\boldsymbol{r}, \Omega_{\mathrm{AT}}\right)}{d_{\mathrm{HY}}}\right] & \boldsymbol{r} \in \Omega_{\mathrm{HY}} \\ 0 & \boldsymbol{r} \in \Omega_{\mathrm{CG}}\end{cases}
$$

where $\operatorname{dist}\left(\boldsymbol{r}, \Omega_{\mathrm{AT}}\right)$ is the distance between the position $\boldsymbol{r}$ and the atomistic region, which is defined by $\operatorname{dist}\left(\boldsymbol{r}, \Omega_{\mathrm{AT}}\right)=\min _{s \in \Omega_{\mathrm{AT}}}|\boldsymbol{r}-\boldsymbol{s}| . d_{\mathrm{HY}}$ is the thickness of the hybrid region. We always assume the thickness of the hybrid region is uniform, which indicates that the weighting function smoothly vanishes at the boundary between the hybrid and the coarse-grained regions. AdResS requires all interactions 24] being treated by the cut-off method, and the thickness of the hybrid region being at least one cut-off radius (denoted by $r_{c}$ ). Moreover, owing to the double-resolution of hybrid molecules, an atomistic molecule only interacts with the atomistic resolution of a hybrid molecule, while a coarse-grained molecule only interacts with the coarse-grained resolution of a hybrid molecule. The atomistic molecules are thus not directly interacting with the coarse-grained molecules. The benefit of these settings is that there is no extra work for modeling the cross-scale interactions.

The intermolecular interactions are well defined in the atomistic and coarse-grained regions by the corresponding models. To setup an AdResS simulation, one only needs to define the interactions in the hybrid region. There are in general two possibilities: force interpolation and the potential interpolation. The force interpolation approach defines the 
hybrid force between two molecules (indexed by $i$ and $j$ ) by a linear interpolation between the atomistic (denoted by $\boldsymbol{F}_{i j}^{\mathrm{AT}}$ ) and coarse-grained (denoted by $\boldsymbol{F}_{i j}^{\mathrm{CG}}$ ) forces.

$$
\boldsymbol{F}_{i j}=w_{i} w_{j} \boldsymbol{F}_{i j}^{\mathrm{AT}}+\left(1-w_{i} w_{j}\right) \boldsymbol{F}_{i j}^{\mathrm{CG}}
$$

where $w_{i}=w\left(\boldsymbol{r}_{i}\right)$ is the weighting function measured at the center-of-mass (COM) position of molecule $i$. It should be noted that the AdResS force interpolation is not conservative, i.e. there does not exist a potential so that the force interpolation is derived by taking the negative gradient of the potential, unless the coarse-grained interaction is identical to the atomistic interaction [18, 19]. It is easy to show that the force interpolation satisfies the momentum conservation: Since both the atomistic and coarse-grained forces are subject to the Newton's third law, i.e. $\boldsymbol{F}_{i j}^{\mathrm{AT}}=-\boldsymbol{F}_{j i}^{\mathrm{AT}}$ and $\boldsymbol{F}_{i j}^{\mathrm{CG}}=-\boldsymbol{F}_{j i}^{\mathrm{CG}}$. From the definition Eq. (2) we have $\boldsymbol{F}_{i j}=-\boldsymbol{F}_{j i}$.

The potential interpolation approach defines the hybrid energy by

$$
V_{i j}=w_{i} w_{j} V_{i j}^{\mathrm{AT}}+\left(1-w_{i} w_{j}\right) V_{i j}^{\mathrm{CG}}
$$

The intermolecular force is therefore calculated by taking the negative gradient on the potential $\boldsymbol{F}_{i j}^{V}=-\nabla_{i} V_{i j}$, which is explicitly written as

$$
\boldsymbol{F}_{i j}^{V}=w_{i} w_{j} \boldsymbol{F}_{i j}^{\mathrm{AT}}+\left(1-w_{i} w_{j}\right) \boldsymbol{F}_{i j}^{\mathrm{CG}}-\nabla w_{i} \cdot w_{j}\left(V_{i j}^{\mathrm{AT}}-V_{i j}^{\mathrm{CG}}\right)
$$

Where $\nabla w_{i}$ is the gradient of the weighting function measured at position $\boldsymbol{r}_{i}$, and the superscript " $V$ " denotes that the force is defined by the potential interpolation. By definition (4), the force of potential interpolation is conservative. The difference between the force and potential interpolations lies in the last term of Eq. (4), which is a force acting along the direction of decreasing weighting function. It should be noted that this force breaks the Newton's third law, therefore the force of potential interpolation does not conserve the momentum. We define the accumulative effect of this term by the force of changing representation:

$$
\boldsymbol{F}_{i}^{\mathrm{rep}}=\sum_{j} \nabla w_{i} \cdot w_{j}\left(V_{i j}^{\mathrm{AT}}-V_{i j}^{\mathrm{CG}}\right)
$$

The fundamental conservation laws satisfied by both the force and potential interpolations are summarized in Tab. I. Unlike normal molecular systems, in which both the momentum and energy are conserved, neither of the AdResS systems conserves both laws. The breaks of these mechanics conservations have substantial influence on the thermodynamic properties 
TABLE I. A summary of the fundamental conservation laws in mechanics satisfied by the normal molecular system and AdResS systems defined by force and potential interpolations.

\begin{tabular}{lcc}
\hline \hline & Momentum Consv. & Energy Consv. \\
Normal molecular System & Yes & Yes \\
AdResS force interpol. & Yes & No \\
AdResS potential interpol. & No & Yes \\
\hline \hline
\end{tabular}

of the AdResS systems (see the discussions in Sec. IV]. Regarding the equilibrium statistical properties, we will show later in Sec. III that both approaches approximately sample the grand-canonical ensemble.

When the intermolecular force is defined by either Eq. (2) or (4), the total force exerts on one molecule is the sum of all pairwise forces:

$$
\boldsymbol{F}_{i}=\sum_{j} \boldsymbol{F}_{i j}, \quad \boldsymbol{F}_{i}^{V}=\sum_{j} \boldsymbol{F}_{i j}^{V}
$$

The force on the atomistic degrees of freedom (DOFs) of a hybrid molecules is distributed by,

$$
\boldsymbol{F}_{\alpha}=\frac{m_{\alpha}}{M_{i}} \boldsymbol{F}_{i}, \quad \boldsymbol{F}_{\alpha}^{V}=\frac{m_{\alpha}}{M_{i}} \boldsymbol{F}_{i}^{V}
$$

where $\alpha$ is the indexes of atoms in molecule $i . m_{\alpha}$ is the mass of atom, and $M_{i}$ is the total mass of molecule $i$, i.e. $M_{i}=\sum_{\alpha \in i} m_{\alpha}$.

Before discussing the statistical and thermodynamic properties of AdResS, a remark on the choice of the weighting function should be added. In Ref. 25] the authors introduced a modified weighing function with a buffer region so that the atomistic region interacts with the hybrid region only via the atomistic intermolecular interaction:

$$
w(\boldsymbol{r})= \begin{cases}1 & \boldsymbol{r} \in \Omega_{\mathrm{AT}} \\ 1 & \boldsymbol{r} \in \Omega_{\mathrm{HY}}, \operatorname{dist}\left(\boldsymbol{r}, \Omega_{\mathrm{AT}}\right)<r_{c} \\ \cos ^{2}\left[\frac{\pi}{2} \cdot \frac{\operatorname{dist}\left(\boldsymbol{r}, \Omega_{\mathrm{AT}}\right)-r_{c}}{d_{\mathrm{HY}}-r_{c}}\right] & \boldsymbol{r} \in \Omega_{\mathrm{HY}}, \operatorname{dist}\left(\boldsymbol{r}, \Omega_{\mathrm{AT}}\right) \geq r_{c} \\ 0 & \boldsymbol{r} \in \Omega_{\mathrm{CG}}\end{cases}
$$

The minimum thickness of the hybrid region is therefore $2 r_{c}$. It worth noting that the coarsegrained region does not require a buffer in the hybrid region, because by definition the coarsegrained region interacts with the hybrid region only via the coarse-grained intermolecular 


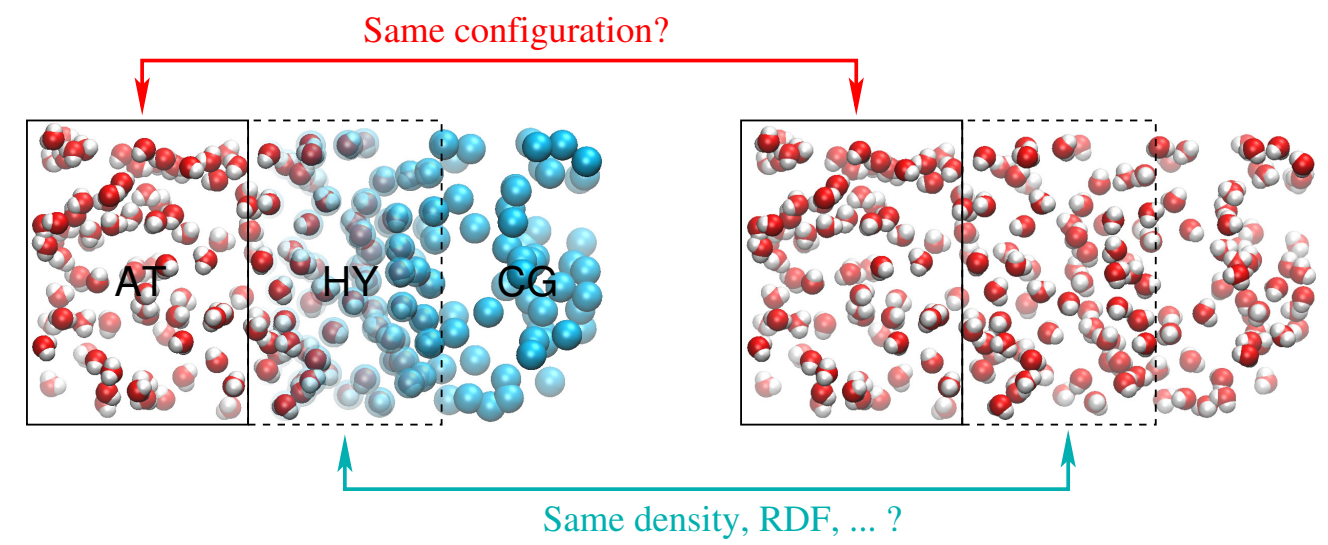

FIG. 2. A schematic plot of the comparison between an AdResS system (left) and the full atomistic reference system (right).

interaction. The new definition (8) is crucial for the equilibrium statistical properties of the AdResS, however, the extra cost is spent in the buffer $\left\{\boldsymbol{r} \mid \boldsymbol{r} \in \Omega_{\mathrm{HY}}\right.$, $\left.\operatorname{dist}\left(\boldsymbol{r}, \Omega_{\mathrm{AT}}\right)<r_{c}\right\}$, which is treated in atomistic resolution. This cost is relatively small for systems with a large atomistic region.

\section{EQUILIBRIUM STATISTICAL PROPERTIES OF ADAPTIVE RESOLU- TION SIMULATION}

In order to consider the accuracy of the AdResS from statistical perspective, we always compare it with a full atomistic reference system, which is of the same size as the AdResS system, and contains the same number of molecules. If the configuration of the atomistic region in the AdResS is same as the corresponding subregion in the atomistic reference, then the AdResS is of good accuracy (see the comparison indicated by the red arrow in Fig. 2). A well-known conclusion of the standard statistical mechanics is that when the reference system approximates the thermodynamic limit, the subsystem is subject to the grand-canonical ensemble. Therefore, it is natural to investigate if the atomistic region of AdResS is also subject to the grand-canonical ensemble in the same thermodynamic limit. The theoretical framework presented in this section works for both the force and potential interpolations.

We denote the number of molecules, volume and temperature of the system by $N, V$ and $T$, respectively. The atomistic reference system has exactly the same set of variables, 
and its equilibrium state is the desired equilibrium of AdResS. The thermodynamic variables of the subregions are specified by the subscript, for example, those of the atomistic region are $N_{\mathrm{AT}}, V_{\mathrm{AT}}$ and $T_{\mathrm{AT}}$. Those of the hybrid and coarse-grained regions are denoted by adding subscripts "HY" and "CG", respectively. Identities $N=N_{\mathrm{AT}}+N_{\mathrm{HY}}+N_{\mathrm{CG}}$ and $V=V_{\mathrm{AT}}+V_{\mathrm{HY}}+V_{\mathrm{CG}}$ obviously hold in the AdResS system. In equilibrium, the temperature is uniform across the system: $T=T_{\mathrm{AT}}=T_{\mathrm{HY}}=T_{\mathrm{CG}}$. This is achieved in practice by coupling the whole system to a Langevin thermostat. The pressure and chemical potential of the atomistic and coarse-grained regions are denoted by $\left\{p_{\mathrm{AT}}, \mu_{\mathrm{AT}}\right\}$ and $\left\{p_{\mathrm{CG}}, \mu_{\mathrm{CG}}\right\}$, respectively. The DOFs of molecules indexed $i$ are $\boldsymbol{x}_{i}=\left\{\boldsymbol{r}_{i}, \boldsymbol{p}_{i}\right\}$, where $\boldsymbol{r}_{i}$ denotes the generalized coordinates and $\boldsymbol{p}_{i}$ denotes the corresponding momenta. All the DOFs of the system are denoted by $\boldsymbol{x}=\left\{\boldsymbol{x}_{1}, \cdots, \boldsymbol{x}_{N}\right\}$. Without lost of generality, we consider that molecules index by $\left\{1, \cdots, N_{\mathrm{AT}}\right\}$ are in the atomistic region, then $\left\{N_{\mathrm{AT}}+1, \cdots, N_{\mathrm{AT}}+N_{\mathrm{HY}}\right\}$ are in the hybrid region, and the last $N_{\mathrm{CG}}$ molecules $\left\{N-N_{\mathrm{CG}}+1, \cdots, N\right\}$ are in the coarsegrained region. The corresponding DOFs are denoted by $\boldsymbol{x}_{\mathrm{AT}}, \boldsymbol{x}_{\mathrm{HY}}$ and $\boldsymbol{x}_{\mathrm{CG}}$. It should be noted that for simplicity, we do not explicitly consider that the number of DOFs are different for an atomistic and a coarse-grained molecule, and uniformly encode them by the vector $\boldsymbol{x}$.

The thermodynamic limit is taken in the sense that both the atomistic and the coarsegrained regions are infinitely large, and at the same time, the atomistic region is much smaller than the coarse-grained region. The hybrid region is much smaller than both the atomistic and coarse-grained regions. Therefore, the volumes of the three regions satisfies $V_{\mathrm{CG}} \gg V_{\mathrm{AT}} \gg V_{\mathrm{HY}}$. The coarse-grained region can be treated as an infinitely large particle and energy reservoir for the atomistic region, and the hybrid region is an infinitely thin filter that changes molecular resolution when a molecule passes by. In the thermodynamic limit, if there were no change of resolution (or considering the full atomistic reference system), it is obvious that the subregion corresponding to the atomistic region samples the grandcanonical ensemble. The question regarding the accuracy of AdResS can be asked by: How accurately the atomistic region samples the grand-canonical ensemble in the thermodynamic limit? More specifically, we want to prove the probability density of the atomistic region satisfies

$$
p\left(\boldsymbol{x}_{\mathrm{AT}}, N_{\mathrm{AT}}\right) \approx \frac{1}{\mathcal{Z}} \exp \left\{\beta \mu_{\mathrm{AT}}^{*} N_{\mathrm{AT}}-\beta \mathcal{H}^{\mathrm{AT}}\left(\boldsymbol{x}_{\mathrm{AT}}\right)\right\}
$$

where $\mu_{\mathrm{AT}}^{*}$ is the chemical potential of reference system in the desired equilibrium, $\mathcal{Z}$ is the 
partition function normalizing the probability density, and $\mathcal{H}^{\mathrm{AT}}$ is the atomistic Hamiltonian.

Instead of directly proving Eq. (9), Ref. 20] suggest investigating the following equivalent equations:

$$
\begin{aligned}
p\left(\boldsymbol{x}_{\mathrm{AT}} \mid N_{\mathrm{AT}}\right) & \approx \frac{1}{Z_{N_{\mathrm{AT}}}} \exp \left\{-\beta \mathcal{H}^{\mathrm{AT}}\left(\boldsymbol{x}_{\mathrm{AT}}\right)\right\} \\
p\left(N_{\mathrm{AT}}\right) & \approx \frac{Z_{N_{\mathrm{AT}}}}{\mathcal{Z}} \exp \left\{\beta \mu_{\mathrm{AT}}^{*} N_{\mathrm{AT}}\right\}
\end{aligned}
$$

where $Z_{N_{\mathrm{AT}}}$ is the canonical partition function for an atomistic system with $N_{\mathrm{AT}}$ molecules

$$
Z_{N_{\mathrm{AT}}}=\int d \boldsymbol{x}_{\mathrm{AT}} \exp \left\{-\beta \mathcal{H}^{\mathrm{AT}}\left(\boldsymbol{x}_{\mathrm{AT}}\right)\right\} \text {. }
$$

The identity of the conditional probability holds: $p\left(\boldsymbol{x}_{\mathrm{AT}}, N_{\mathrm{AT}}\right)=p\left(\boldsymbol{x}_{\mathrm{AT}} \mid N_{\mathrm{AT}}\right) p\left(N_{\mathrm{AT}}\right)$.

\section{A. The accuracy of the configurational probability density}

The configurational probability density 10 is further split as

$$
p\left(\boldsymbol{x}_{\mathrm{AT}} \mid N_{\mathrm{AT}}\right)=\sum_{N_{\mathrm{HY}}} \int d \boldsymbol{x}_{\mathrm{HY}} p\left(\boldsymbol{x}_{\mathrm{AT}} \mid N_{\mathrm{AT}} ; \boldsymbol{x}_{\mathrm{HY}}, N_{\mathrm{HY}}\right) \cdot p\left(\boldsymbol{x}_{\mathrm{HY}}, N_{\mathrm{HY}} \mid N_{\mathrm{AT}}\right)
$$

The first probability density in the integral is the probability density of atomistic DOFs conditioned on the number of atomistic molecules and all hybrid DOFs. It can be shown that if (1) All interactions in the system are cut-offed; (2) The atomistic region interacts with the hybrid region only in an atomistic way; (3) The system is short-range correlated, and the correlation between the atomistic and the coarse-grained regions is negligible, then the probability density $p\left(\boldsymbol{x}_{\mathrm{AT}} \mid N_{\mathrm{AT}} ; \boldsymbol{x}_{\mathrm{HY}}, N_{\mathrm{HY}}\right)$ is approximated by

$$
p\left(\boldsymbol{x}_{\mathrm{AT}} \mid N_{\mathrm{AT}} ; \boldsymbol{x}_{\mathrm{HY}}, N_{\mathrm{HY}}\right) \propto \exp \left\{-\beta \mathcal{H}^{\mathrm{AT}}\left(\boldsymbol{x}_{\mathrm{AT}} ; \boldsymbol{x}_{\mathrm{HY}}, N_{\mathrm{HY}}\right)\right\}
$$

where

$$
\mathcal{H}^{\mathrm{AT}}\left(\boldsymbol{x}_{\mathrm{AT}} ; \boldsymbol{x}_{\mathrm{HY}}, N_{\mathrm{HY}}\right)=\sum_{i=1}^{N_{\mathrm{AT}}} \frac{1}{2} m_{i} \boldsymbol{v}_{i}^{2}+\sum_{i, j=1}^{N_{\mathrm{AT}}} \frac{1}{2} V^{\mathrm{AT}}\left(\boldsymbol{r}_{i j}\right)+\sum_{i=1}^{N_{\mathrm{AT}}} \sum_{j=N_{\mathrm{AT}}+1}^{N_{\mathrm{AT}}+N_{\mathrm{HY}}} V^{\mathrm{AT}}\left(\boldsymbol{r}_{i j}\right)
$$

is the atomistic Hamiltonian with parameters $\boldsymbol{x}_{\mathrm{HY}}, N_{\mathrm{HY}}$. It should be noted that the probability density (14) is identical to that of the reference system. The probability density and local Hamiltonian can be written down for the coarse-grained region analogically. 
In general, the second probability density $p\left(\boldsymbol{x}_{\mathrm{HY}}, N_{\mathrm{HY}} \mid N_{\mathrm{AT}}\right)$ in Eq. (13) is not the same as the reference system. For example, it has been shown that the density distribution in the hybrid region deviates from the desired one, even if the coarse-grained side is modeled to reproduce the atomistic pressure [6]. However, it is possible to raise necessary conditions for the AdResS system, with which the accuracy can be improved systematically (see the cyan arrow in Fig. 2). The first necessary condition is that the density profile of the hybrid region should be a constant that is identical to the density of the atomistic reference at desired equilibrium:

$$
\rho_{\mathrm{HY}}(\boldsymbol{r})=\rho_{\mathrm{AT}}^{*}=\frac{N}{V}
$$

The second necessary condition is that the two body probability density in the hybrid region is the same as the full atomistic reference, In homogeneous and isotropic system, it is equivalent to ask that the hybrid radial distribution function (RDF) should be the same as that of the reference system:

$$
g_{\mathrm{HY}}(r)=g_{\mathrm{AT}}^{*}(r) .
$$

It is fully justified to systematically raise the necessary conditions up to $m$-th multibody probability density, for example the third necessary condition would be

$$
C_{\mathrm{HY}}^{(3)}=C_{\mathrm{AT}}^{(3) *}
$$

where $C^{(3)}$ is the three-body correlation. Since the system is homogeneous and isotropic, the three-body probability density is equivalent to three-body correlation function.

In practice, the density in the hybrid region is corrected by the thermodynamic force [8], which is applied on top of the AdResS intermolecular interactions:

$$
\begin{gathered}
\boldsymbol{F}_{i}=\sum_{j} \boldsymbol{F}_{i j}+\boldsymbol{F}_{i}^{\mathrm{th}}, \\
\boldsymbol{F}_{i}^{V}=\sum_{j} \boldsymbol{F}_{i j}^{V}+\boldsymbol{F}_{i}^{\mathrm{th}, V},
\end{gathered}
$$

where the thermodynamic forces is a one-body force defined over space, i.e. $\boldsymbol{F}_{i}^{\text {th }}=\boldsymbol{F}^{\text {th }}\left(\boldsymbol{r}_{i}\right)$ and $\boldsymbol{F}_{i}^{\mathrm{th}, V}=\boldsymbol{F}^{\mathrm{th}, V}\left(\boldsymbol{r}_{i}\right)$, for force and potential interpolations, respectively. The thermodynamic force is applied only in the hybrid region, and is calculated by the following iterative scheme:

$$
\boldsymbol{F}_{k+1}^{\mathrm{th}(, V)}(\boldsymbol{r})=\boldsymbol{F}_{k}^{\mathrm{th}(, V)}(\boldsymbol{r})-\frac{M}{\kappa\left(\rho_{\mathrm{AT}}^{*}\right)^{2}} \nabla \rho_{k}(\boldsymbol{r})
$$


where $k$ denotes the step of iteration and $\kappa$ is the isothermal-compressibility. In equilibrium the density profile $\rho(\boldsymbol{r})$ in the atomistic and coarse-grained regions are constants, so the thermodynamic force is updated only in the hybrid region. When the hybrid density is flat, the thermodynamic force converges. An important consequence is that

$$
\rho_{\mathrm{AT}}(\boldsymbol{r})=\rho_{\mathrm{HY}}(\boldsymbol{r})=\rho_{\mathrm{CG}}(\boldsymbol{r})=\rho_{\mathrm{AT}}^{*},
$$

because the density profiles match at the atomistic-hybrid and hybrid-coarse-grained boundaries.

By using a numerical example of SPC/E water [26] system, it has been shown in Ref. [20] that when the thermodynamic force is applied, the AdResS atomistic region reproduces (with satisfactory accuracy) multi-body configurational probability densities of the reference system up to the three-body correlation. A surprising numerical observation is that although only the thermodynamic force is applied, the second order necessary condition (17) is automatically fulfilled, because the RDF is identical to the reference system in the buffer hybrid region $\left\{\boldsymbol{r}: \boldsymbol{r} \in \Omega_{\mathrm{HY}}\right.$, $\left.\operatorname{dist}\left(\boldsymbol{r}, \Omega_{\mathrm{AT}}\right)<r_{c}\right\}$ that is interacting with the atomistic region. The third necessary condition is not satisfied because there is deviation in the three-body correlation in the buffer hybrid region. It should be noted that there is no theoretical background why the second necessary condition is satisfied by only using the thermodynamic force, so one cannot expect the same benefit in other systems.

The second necessary condition $(17)$ is fulfilled by the RDF correction $\boldsymbol{F}^{\text {rdf }}$ [25], which is a conservative two-body force added to the force interpolation (2) as an extra term that applies only in the hybrid region:

$$
\boldsymbol{F}_{i j}=w_{i} w_{j} \boldsymbol{F}_{i j}^{\mathrm{AT}}+\left(1-w_{i} w_{j}\right) \boldsymbol{F}_{i j}^{\mathrm{CG}}+w_{i} w_{j}\left(1-w_{i} w_{j}\right) \boldsymbol{F}_{i j}^{\mathrm{rdf}}
$$

The RDF correction force is constructed from a RDF correction potential $V^{\text {rdf }}$ by

$$
\boldsymbol{F}_{i j}^{\mathrm{rdf}}=\boldsymbol{F}^{\mathrm{rdf}}\left(\boldsymbol{r}_{i j}\right)=-\nabla_{\boldsymbol{r}} V^{\mathrm{rdf}}\left(r_{i j}\right)
$$

which is calculated by the iterative Boltzmann inversion:

$$
V_{k+1}^{\mathrm{rdf}}(r)=V_{k}^{\mathrm{rdf}}(r)+k_{B} T \ln \left[\frac{g_{k}(r)}{g_{\mathrm{AT}}^{*}(r)}\right]
$$

where $k$ is the step of iteration. When the RDF of $k$ th iteration is identical to that of the full atomistic reference $g_{\mathrm{AT}}^{*}(r)$, the iteration converges. It has been shown that the iterative 
scheme of thermodynamic force (21) coupled with the iterative Boltzmann inversion (25) is effective in correcting the density profile $g_{\mathrm{HY}}(\boldsymbol{r})$ and RDF $g_{\mathrm{HY}}(r)$ simultaneously [25]. For $\mathrm{SPC} / \mathrm{E}$ water system when the RDF correction is applied, the third necessary condition is automatically satisfied [20]. Again, there is no theoretical prove behind this phenomenon, and one cannot expect the same benefit for other systems.

Remark: The RDF correction is developed only for the force interpolation scheme, and a natural extension to the potential interpolation seems to be

$$
V_{i j}=w_{i} w_{j} V_{i j}^{\mathrm{AT}}+\left(1-w_{i} w_{j}\right) V_{i j}^{\mathrm{CG}}+w_{i} w_{j}\left(1-w_{i} w_{j}\right) V_{i j}^{\mathrm{rdf}}
$$

The effectiveness of this proposal has never been tested, and is still an open problem.

\section{B. The accuracy of the number probability}

The accuracy of number probability $p\left(N_{\mathrm{AT}}\right)$ is investigated by a Taylor expansion w.r.t. the relative size of the atomistic region to the whole system, which vanishes in the thermodynamic limit. The sufficient condition for the first order accuracy asks for a balance of the chemical potential [20]:

$$
\mu_{\mathrm{CG}}-\mu_{\mathrm{AT}}=\omega_{0}
$$

where $\omega_{0}$ corresponds to the work from the filter on each molecule that leaves the coarsegrained region and enters the atomistic region. An important conclusion form Ref. [20] is that the relation (27) holds automatically when the thermodynamic force is applied so that the atomistic and coarse-grained densities match. The conclusion is derived under the assumption of thermodynamic limit and the decorrelation between the atomistic and coarse-grained regions.

The second order accuracy is achieved if the isothermal-compressibility of the atomistic and coarse-grained resolutions match:

$$
\kappa_{\mathrm{AT}}=\kappa_{\mathrm{CG}}
$$

This is achieved by coarse-grained modeling, for example, the structure based coarse-grained models that reproduce the atomistic RDF [27]. 


\section{The WCA potential as a generic energy and particle reservoir}

From the analysis on statistical mechanics, one surprising fact is that if the requirement for the accuracy is not very high (only applying the thermodynamic force is acceptable), then there is actually no restriction on the coarse-grained model. One can even use ideal gas as a coarse-grained model. The computational difficulty lies in the sudden switching-on of the atomistic interactions when a coarse-grained molecule enters the hybrid region. If two molecules enter at the same time and the same location, then the atomistic contribution to the intermolecular interaction is infinity, which drives the simulation unstable. This numerical difficulty can be avoided by using capped atomistic interaction [1], or by using a gradually switching-on core-softened atomistic interaction [14]. In Ref. [20], the authors instead tested with Weeks-Chandler-Andersen (WCA) potential [28] as the coarse-grained model, which is a short-ranged and pure repulsive interaction. For an SPC/E water system, the cut-off radius of WCA potential can be 2.4 time smaller than the cut-off radius used in the atomistic region. Since the computational cost of cut-off scheme is of order $\mathcal{O}\left(r_{c}^{3}\right)$, the pair interaction of WCA is 19 times cheaper. Moreover, for each pair of molecules the atomistic model computes 10 pairwise interactions (9 electrostatic +1 van der Waals), while the WCA model computes only one. Therefore the WCA model in total costs only 1/190 on force computation than the SPC/E atomistic model. The WCA approach has been successfully used in calculating the chemical potential of various complex fluids and mixtures [22].

\section{THERMODYNAMIC PROPERTIES OF ADAPTIVE RESOLUTION SIMU- LATION}

In the reference system (full atomistic), if the equilibrium state is achieved, then the temperature, pressure and chemical potential of different regions are identical. In AdResS system, the global uniform temperature distribution in equilibrium is equilibrated by the Langevin thermostat. However, extra terms appear in the pressure or chemical potential relations when the momentum or energy conservation is absent (see Tab. If), respectively.

The chemical potential balance can be investigated from the fact that when the uniform density distribution is guaranteed, the chemical potential difference between the resolutions 
is compensated by $\omega_{0}$, which is the work per molecule from the filter when a molecule leaves the coarse-grained region and enters the atomistic region (see relation (27)). Now the question turns out to be what contributes to the work $\omega_{0}$. For the potential interpolation, the work of the filter is nothing but the work of the thermodynamic force plus the extra kinetic energy corresponding to the extra DOFs that a molecule obtains in higher resolution region:

$$
\omega_{0}=\omega^{\mathrm{th}, V}+\omega^{\mathrm{DOF}}
$$

where $\omega^{\text {th }, V}$ denotes the work of the thermodynamic force of the potential interpolation. This work is path independent if the thermodynamic force is defined as a function of the distance to the atomistic region, and applies along the gradient of the weighting function. We always use the thermodynamic force that satisfies these conditions. For the force interpolation, we denote the extra contribution due to the absence of the energy conservation by $\omega^{\text {extra }}$, then

$$
\omega_{0}=\omega^{\text {th }}+\omega^{\text {extra }}+\omega^{\mathrm{DOF}},
$$

where $\omega^{\text {th }}$ is the work of the thermodynamic force of the force interpolation. The magnitude of the extra term $\omega^{\text {extra }}$ is unclear up to now.

For the force interpolation, the pressure of different resolutions is related by [8]

$$
p_{\mathrm{CG}}-p_{\mathrm{AT}}=\rho_{0} \omega^{\mathrm{th}}
$$

in which the work of the thermodynamic force again plays an important role. For the potential interpolation, the pressure relation can be investigated by an imaginary infinitesimal volume increment of the atomistic region, and the same amount of volume decrement of the coarse-grained region. Following this idea, Ref. [22] proves that in equilibrium the pressure is related by

$$
p_{\mathrm{CG}}-p_{\mathrm{AT}}=\rho_{0}\left(\omega^{\mathrm{th}, V}-\omega^{\mathrm{rep}}\right)
$$

where $\omega^{\text {rep }}$ is the work done by the average force of changing representation:

$$
\omega^{\mathrm{rep}}=\int\left\langle\boldsymbol{F}^{\mathrm{rep}}(\boldsymbol{r})\right\rangle_{V} \circ d \boldsymbol{r}
$$

where "o" means that the integral should be understood as along the path. The force of changing representation acts always along the gradient of the weighting function, and the 
TABLE II. A summary of the thermodynamic relations satisfied in force and potential interpolation AdResS.

Force interpol. AdResS

Temperature $\quad T_{\mathrm{AT}}=T_{\mathrm{CG}}$

Pressure

$$
p_{\mathrm{CG}}-p_{\mathrm{AT}}=\rho_{0} \omega^{\mathrm{th}}
$$

Chemical pot.

$\mu_{\mathrm{CG}}-\mu_{\mathrm{AT}}=\left(\omega^{\mathrm{th}}+\omega^{\mathrm{DOF}}+\omega^{\mathrm{rep}}\right)$

weighting function is a function of the distance to the atomistic region, therefore, if the system is homogeneous in the hybrid region, then the integral (33) is path independent. The ensemble average can be estimated by sampling. The force of changing representation stems from the last term of potential interpolation (4), and contributes to the pressure relation (32) because it does not satisfy the Newton's action-reaction law [22].

Using Eq. (29) and (30) one has $\omega^{\text {th }, V}-\omega^{\text {th }}=\omega^{\text {extra }}$, while using (31) and (32) one has $\omega^{\text {th }, V}-\omega^{\text {th }}=\omega^{\text {rep }}$. Therefore, we have

$$
\omega^{\text {extra }}=\omega^{\text {rep }}
$$

It should be noted that the ensemble average in the work of changing representation (33) is defined for the potential interpolation, however, the estimate of the term $\omega^{\text {rep }}$ does not depend on the ensemble, at least up to the "first order approximation" [22], because the hybrid region of the potential and force interpolation have the same density. In Ref. [20] an even stronger conclusion is shown numerically in the SPC/E water system: The average force of changing representation estimated from potential interpolation is pointwisly identical to that estimated from the force interpolation. The thermodynamic relations discovered in force and potential interpolation AdResS schemes are summarized in Tab. II.

\section{DYNAMICAL PROPERTIES: ADAPTIVE RESOLUTION SIMULATION BE- YOND EQUILIBRIUM}

In principle, all quantities that can be written down in terms of equilibrium ensemble averages should be computed with high accuracy when using an AdResS simulation that approximately samples the grand-canonical ensemble. However, under this framework one has little knowledge regarding the quantities that are not ensemble averages. For example, 
the non-equilibrium response of the system with respect to an external perturbation, which is either computed by brute force non-equilibrium simulations [29], or by equilibrium timecorrelation function via the Green-Kubo relation [30, 31] if the perturbation is small. The time-correlation function, although measured in equilibrium simulations, depends on how the dynamics of the system is implemented. The quantities of this type are called dynamical properties of the system. In principle, the Hamiltonian dynamics should be used to compute the dynamical properties, however, due to the truncation error in computer simulations, it is technically difficult to conserve the energy in very long MD simulations. To this end thermostats are sometimes coupled to keep the system at desired thermodynamic state. It is worth noting that the thermostats are designed to sample equilibrium distributions, and disturb the Hamiltonian dynamics in an artificial way. Therefore, when applying the thermostats in computing the dynamical properties, the potential artifacts should be checked carefully. In the context of AdResS, we propose an alternative way of thermostating the system, the local thermostat method [29], which is proved to be free of the artifacts in computing the dynamical properties, in the following section.

\section{A. Velocity auto-correlation of water}

In this section we take the normalized velocity auto-correlation function for example:

$$
C_{v}(t)=\frac{\langle\boldsymbol{v}(0) \cdot \boldsymbol{v}(t)\rangle}{\langle\boldsymbol{v}(0) \cdot \boldsymbol{v}(0)\rangle}
$$

where the averages $\langle\cdot\rangle$ are still taken with respect to the equilibrium ensemble, and the term $\langle\boldsymbol{v}(0) \cdot \boldsymbol{v}(t)\rangle$ computes the correlation between the velocity at time 0 and $t$, which depends on the dynamics of the system. An illustrative example of the thermostating artifact is the oxygen velocity auto-correlation in a TIP3P [32] water system presented in Fig. 3. If the system is coupled to a Langevin thermostat, the result is qualitatively different from that without any thermostat coupling, i.e. Hamiltonian dynamics. Although by using milder thermostats (increased time-scales) will improve the result, it is in general difficult to know a priori the strength of the thermostat coupling. The standard AdResS simulations, since the Langevin thermostat is globally coupled to the system, is not suitable for reproducing the dynamical properties of the system.

The solution is to adopt the local thermostat method that was initially developed for non- 


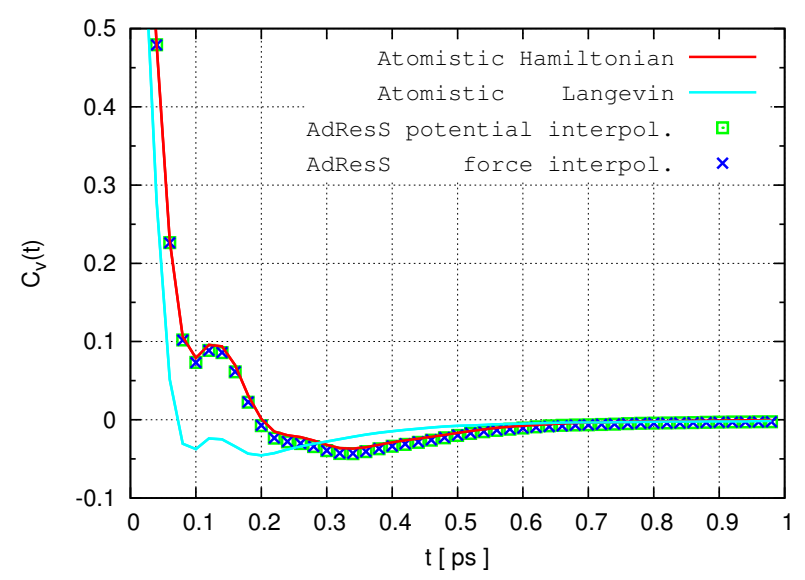

FIG. 3. The oxygen velocity auto-correlation by atomistic simulation compared with AdResS simulation. The solid cyan and red lines compares the atomistic simulation with and without Langevin thermostat, respectively. In the simulation of Hamiltonian dynamics, the energy drift is only $0.01 \%$. The square and cross points represent the potential and force interpolation AdResS with local thermostat, respectively. The time-scales of the global and local Langevin thermostat are both $\tau_{T}=0.1 \mathrm{ps}$.

equilibrium MD simulations [29]. This method divides the system into two regions. The dynamical region, out of which the dynamical properties are calculated, is not coupled to any thermostat, so the dynamics there is preserved as Hamiltonian dynamics. The thermostated region is coupled to the Langevin thermostat in order to keep the system at the desired thermodynamic state. In the context of AdResS, we let the dynamical region identical to the atomistic region, and the hybrid and coarse-grained regions, which serve as particle and energy reservoir, coupled to the thermostat.

This local thermostat method is validated by an AdResS simulation of pure TIP3P water system. The simulation is performed by the Gromacs [33] version 4.6.5. The system contains 5000 water molecules. The dimension of the periodic simulation region is $14.7 \times 3.2 \times 3.2 \mathrm{~nm}^{3}$, in which the resolution changes only in $x$-direction. The atomistic region is of size $1.5 \times$ $3.2 \times 3.2 \mathrm{~nm}^{3}$, and the width of the hybrid region is $d_{\mathrm{HY}}=2.85 \mathrm{~nm}$. The rest of the system is coarse-grained region modeled by the WCA interaction. The weighting function of form Eq. (8) is used to couple different resolutions. The Langevin thermostat coupled to the hybrid and coarse-grained regions is of time-scale $\tau_{T}=0.1 \mathrm{ps}$. The thermodynamic forces of force and potential interpolations are iteratively computed by Eq. (21). The electrostatic 
(a)

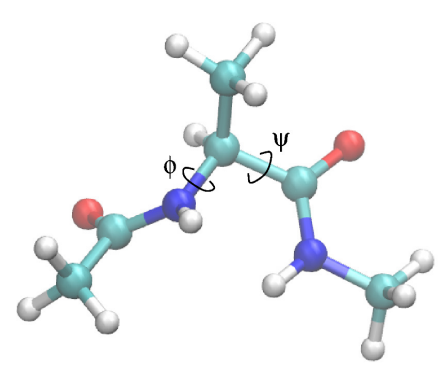

(b)

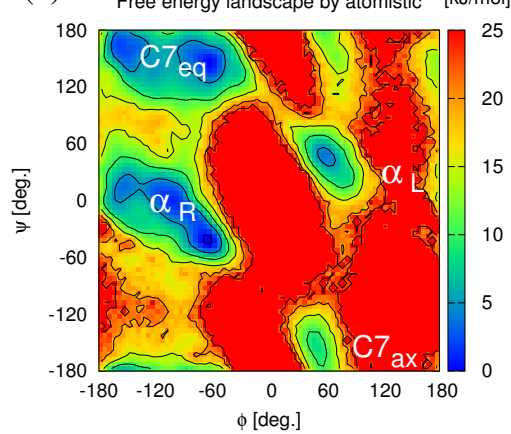

(c) Free energy landscape by AdResS [kJ/mol]

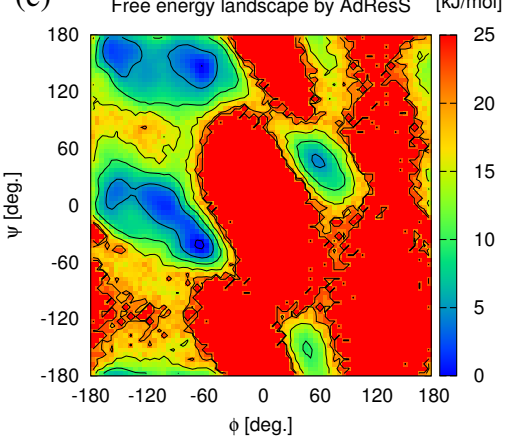

FIG. 4. (a): A schematic plot of the alanine dipeptide molecule, and the two dihedral angles $\phi$ and $\psi$ that are used to represent the conformations of the molecule. (b) and (c): The free energy landscape plotted using variables $\phi$ and $\psi$. (b) is for full atomistic reference simulation, while (c) is for AdResS. The metastable conformations coded by $\alpha_{\mathrm{R}}, \mathrm{C} 7_{\text {eq }}, \alpha_{\mathrm{L}}$ and $\mathrm{C} 7_{\mathrm{ax}}$ are noted on plot (b).

interaction is treated by the Reaction-Field method [34, 35], with dielectric constant $\varepsilon_{\mathrm{rf}}=$ $+\infty$, which conserves the energy, and is proved to be a special case of the Zero-multiple method [36, 37]. The cut-off radius is $1.2 \mathrm{~nm}$, and the van der Waals interaction is smoothed by the "switch" method provided by Gromacs.

The Fig. 3 presents the velocity auto-correlation functions computed by fully equilibrated MD trajectories of length $1 \mathrm{~ns}$, with the velocities recorded every 0.04 ps. Here full equilibration means uniform temperature and density distributions in the system. In the Figure, both the force and potential interpolations are in almost perfect agreement with the atomistic simulation under Hamiltonian dynamics. This indicates that AdResS with local thermostat is a promising method to investigate the dynamical properties in the concurrent multiscale simulation.

\section{B. Alanine dipeptide dissolved in water: equilibrium and dynamical properties}

The alanine dipeptide molecule (see Fig. 4(a)) is usually used as a testing case for evaluating the new computational method in simulating biomolecular system (see e.g. Ref. [38 41]). We investigate a system composed of 7235 TIP3P water molecules, and one alanine dipeptide described by the CHARMM27 force field [42] with grid-based energy correction map 
(CMAP) [43]. The dimension of the simulation region is $6.1 \times 6.1 \times 6.1 \mathrm{~nm}^{3}$, which is divided into a spherical atomistic region of radius $0.5 \mathrm{~nm}$ centered at the $\alpha$-carbon and a shell-shaped hybrid region of width $2.0 \mathrm{~nm}$. The rest of the system is treated coarse-grainly by the WCA interaction. The dynamical region is also centered at the $\alpha$-carbon, and is of radius $1.2 \mathrm{~nm}$. It has been proved that the conformational dynamics of alanine dipeptide is not sensitive to the size of the dynamical region when the radius is larger than $1.0 \mathrm{~nm}$ [29]. In this case, part of the hybrid region is not thermostated, so when using force interpolation, the extra energy production might not be effectively equilibriated. To this end, we only investigate the potential interpolation. The corresponding thermodynamic force is iteratively computed by Eq. (21). The electrostatic interaction is computed by the Reaction-Field method with dielectric constant $\varepsilon_{\mathrm{rf}}=+\infty$. Both the electrostatic and the van der Waals interactions (smoothed by "switch" method in Gromacs) are cut-offed at $1.0 \mathrm{~nm}$.

The system presents a very clear time-scale separation: There are fast motions like bond and angle vibrations that happen in tens of femtoseconds, while there are slow conformational transitions that happen in hundreds of picoseconds. The dihedral angles $\phi$ and $\psi$ (see Fig. $4($ a) ) are usually chosen as collective variables that describe the molecular conformation and its transitions.

The free energy is defined as the logarithm of the projected equilibrium probability density on the $\phi-\psi$ space:

$$
F(\phi, \psi)=-k_{B} T \ln p_{\text {eq }}(\phi, \psi)
$$

The free energy of the full atomistic reference system is showed in Fig. 4 (b), which clearly presents the metastability in the system: several highly populated regions that correspond to metastable conformations are separated by lowly populated regions that are identified as transition regions. The AdResS free energy (Fig. 4 (c)) is in good consistency with the full atomistic reference system, and reproduces the metastable conformations and their populations with satisfactory accuracy. This is expected in equilibrium, because as discussed in Sec. III, the AdResS sampling approximates the atomistic grand-canonical ensemble.

In practice, not only the equilibrium properties like the free energy are of interest, but also the dynamical properties like the conformational transitions and the corresponding relaxation time-scales are of highly biological importance. When the system presents metastability, one powerful tool for this purpose is the Markov state model (MSM) [44 46], which 
approximates the original high-dimensional dynamics by a finite-state reduced Markov process. The accuracy analysis regarding this approximation is well-established [47, 48]. The MSM has found successfully applications in various fields, for example studying complex protein systems (two of the hundreds of examples are Ref. [49, 50]).

In a standard MSM approach, the full phase space is firstly decomposed into a nonoverlapping union of finite number of states (not necessarily identical to the metastable conformations), then the original time-dependent probability density define on the phase space $\left(p_{t}(\boldsymbol{x})\right)$ is approximated by a temporal discrete probability $\boldsymbol{p}_{k \tau}$ defined on the finitestate space. The step of the temporal discretization $\tau$ is called the lag-time. The reduced dynamics is assumed to be Markovian. Under the assumption of Markovianity, the original continuous dynamics is approximated by the Markov processes governed by

$$
\boldsymbol{p}_{k \tau}^{\top}=\boldsymbol{p}_{0}^{\top} \cdot \boldsymbol{T}^{k}(\tau)
$$

where $\boldsymbol{T}(\tau)$ is the transition matrix that associates to the reduced dynamics of $\boldsymbol{p}_{k \tau}^{\top}$. The matrix element, for example $T_{i j}$ is defined as the probability of the system being in state $j$ at $\tau$, provided the system being in state $i$ at time 0 . In equilibrium, the transition matrix $\boldsymbol{T}(\tau)$ does not depend on time when the state $i$ is investigated, and only the lag-time $\tau$ matters. If the transition matrix is irreducible and aperiodic, then according to Perron-Frobenius theorem, the maximum eigenvalue is reached and single. Due to the stochasticity of the matrix, this eigenvalue is equal to 1 . If the dynamics is further assumed to be reversible, then all eigenvalues of the transition matrix are real valued. The first $m$ largest eigenvalues are denoted by $1=\lambda_{1}>\lambda_{2} \geq \lambda_{3} \geq \cdots \geq \lambda_{m}$, and the corresponding left eigenvectors are denoted by $\Phi_{1}, \Phi_{2}, \cdots, \Phi_{m}$. The leading eigenvector is non-negative, and satisfies $\Phi_{1}^{\top}=$ $\Phi_{1}^{\top} \cdot \boldsymbol{T}(\tau)$. With proper normalization (integrate to 1 ) the leading eigenvector is identical to the equilibrium probability density. At infinitely long time, the probability density $\boldsymbol{p}_{k \tau}$ converges to the equilibrium probability. The speed of the convergence is characterized by the leading relaxation time-scales that are determined by the leading eigenvalues: $t_{i}=$ $-\tau / \log \lambda_{i}, i=2, \cdots, m$. Since the eigenvectors are orthogonal and the first eigenvector is non-negative, the reset of the eigenvectors have a vanished sum over all elements: Their value is positive at some states and negative at some other states. The different signs in the eigenvectors denote the conformational transition corresponding to the time-scale $t_{i}$.

In the example of alanine dipeptide dissolved in water, the states in the MSM is defined 


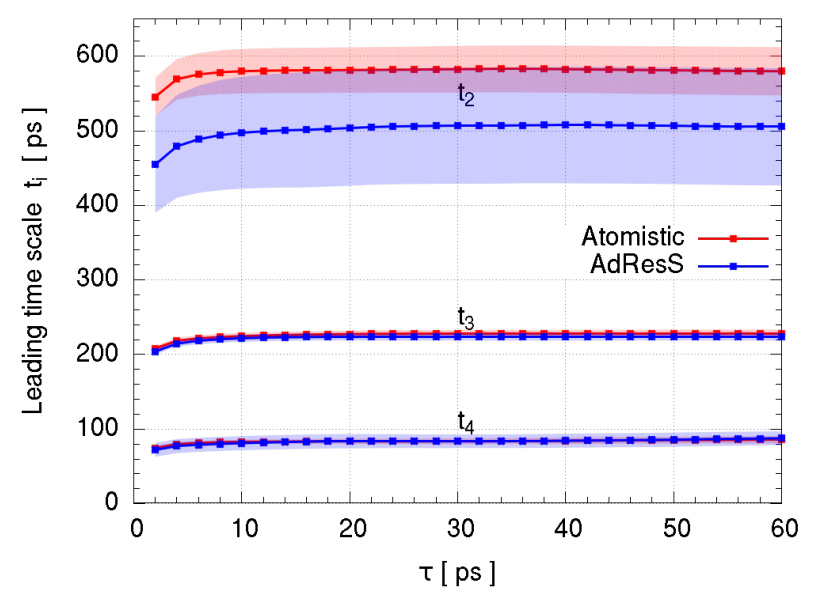

FIG. 5. The non-trivial leading time-scale calculated from the Markov state modeling for the system of alanine dipeptide dissolved in water. In this figure, $t_{2} t_{3}$ and $t_{4}$ correspond to the first, second and third non-trivial leading time-scale, respectively. The horizontal axis $\tau$ is the lag-time used to build the Markov state model. For a sufficient large $\tau$ the values of the time-scales should be independent on the lag-time. The shadowing regions around the lines indicate the statistical uncertainty of the result.

by uniformly dividing the $\phi-\psi$ space in to $20 \times 20$ bins. Each bin presents a state in MSM, and the transition matrix is sampled by equilibrium MD simulations with the local thermostating scheme [29]. The MSM is built and analyzed by the software EMMA [51]. The resulting eigenvalues and eigenvectors of AdResS are compared with the full atomistic reference under the same local thermostat in Fig. 5 and 6. The eigenvalues should be independent for sufficiently large $\tau$ if the MSM is of good accuracy, and from Fig. 5 they are so for $\tau \geq 20$ ps. Also from the Fig. 5, the full atomistic reference and the AdResS eigenvalues are consistent within the statistical uncertainty. From Fig. 6, the conformational transitions correspond to $t_{2}, t_{3}$ and $t_{4}$ are $\left\{\alpha_{\mathrm{R}}\right\} \leftrightarrow\left\{\alpha_{\mathrm{L}}, \mathrm{C} 7_{\mathrm{ax}}\right\},\left\{\alpha_{\mathrm{R}}, \alpha_{\mathrm{L}}\right\} \leftrightarrow\left\{\mathrm{C} 7_{\text {eq }}\right\}$ and $\left\{\alpha_{\mathrm{L}}\right\} \leftrightarrow$ $\left\{\mathrm{C}_{\mathrm{ax}}\right\}$, respectively. The eigenvectors and the indicated conformational transitions of the AdResS are also consistent with those of the full atomistic reference. Although calculated from the equilibrium MD simulations, it should be noted that the leading time-scales and the corresponding eigenvectors are not the equilibrium properties, because the transition probabilities (elements of the transition matrix) are actually auto-correlation functions of the characteristic functions that are not equilibrium ensemble averages. 

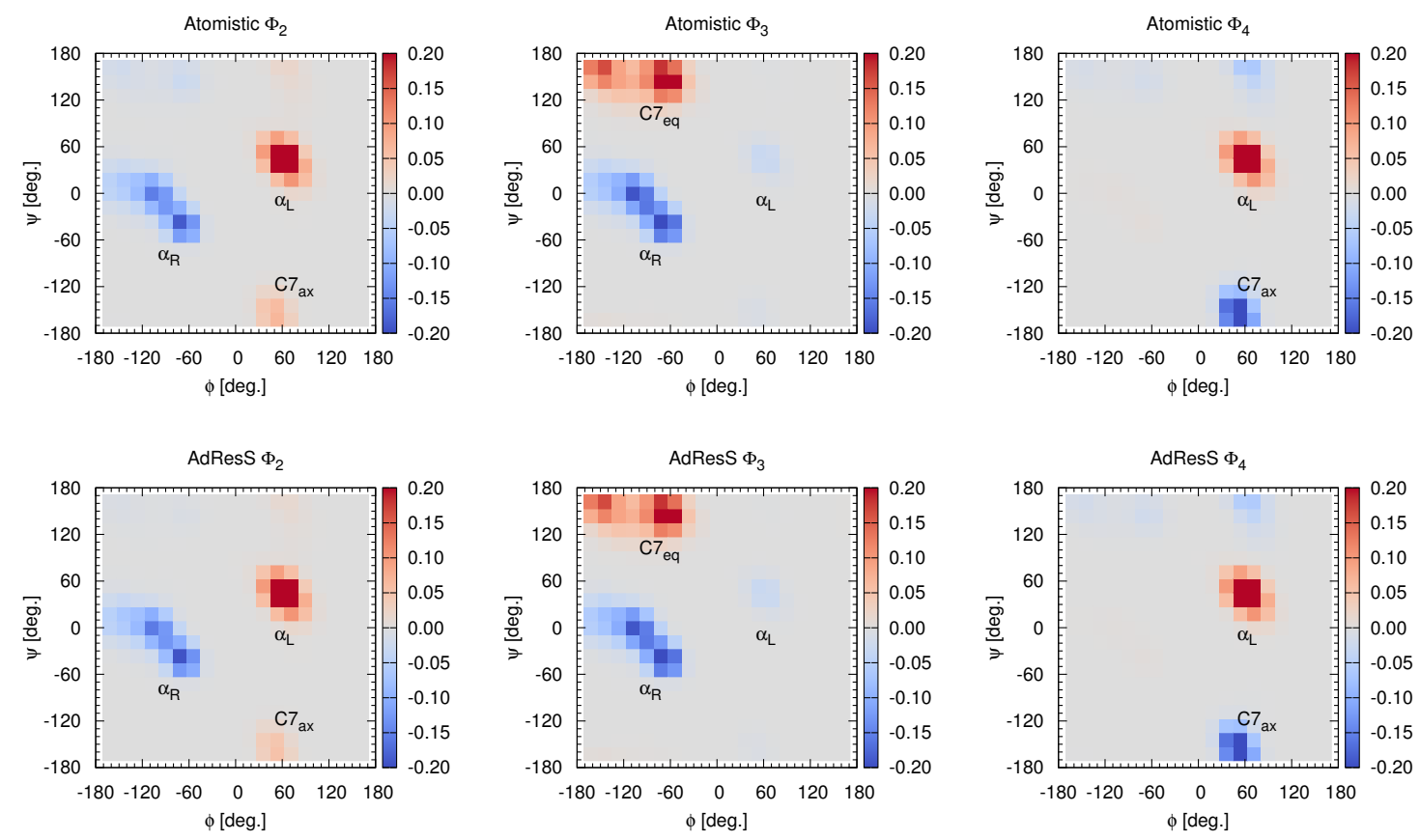

FIG. 6. The eigenvectors corresponding to the first three leading time-scales computed using lagtime 20 ps. The first row represents the eigenvectors of the full atomistic reference, while the second row represents those of the AdResS. From left to right $\Phi_{2}, \Phi_{3}$ and $\Phi_{4}$ that correspond to time-scales $t_{2}, t_{3}$ and $t_{4}$ are given (see also Fig. 5). The magnitude of the eigenvectors are denoted by the color. The conformational changes indicated by the eigenvectors are noted on the plots.

\section{CONCLUDING REMARKS AND OPEN QUESTIONS}

In this paper, we investigated both the potential and force interpolations under a uniform theoretical framework of GC-AdResS. The necessary conditions for the grand-canonical like equilibrium simulations are provided. However, it should be noticed that these conditions are not sufficient, and the high accuracy in the numerical simulations indicates that these conditions tend to be too "strong". Take the AdResS water simulation for example, when only the thermodynamic force is applied to impose the first necessary condition (density consistency), the configuration of the atomistic region is almost identical to the full atomistic reference up to the three-body correlation [52], and the second necessary condition (RDF consistency) is automatically fulfilled. It might also be possible that the errors in the numerical simulations are too small to be observed. In this context, it would be helpful 
to provide error estimates on the ensemble sampled by AdResS with respect to the full atomistic grand-canonical ensemble. To the knowledge of the authors, neither the sufficient condition nor the error estimate has ever been investigated.

If one were able to answer the questions regarding the accuracy of the equilibrium ensemble of AdResS, the accuracy of quantities that are equilibrium ensemble averages would be automatically answered. However, the dynamical properties, which are not equilibrium ensemble averages, are out of the scope of the GC-AdResS framework. In order to numerically check the accuracy of AdResS in computing the dynamical properties, we investigated the velocity auto-correlation function of pure water and the leading relaxation time-scales of alanine dipeptide dissolved in water. The significant time-scale of the time-correlations ranges from 1 ps in the case of velocity auto-correlation to almost 600 ps in the case of conformational relaxation of alanine dipeptide. In both systems the AdResS reproduces the atomistic result with a satisfactory agreement. Although these positive numerically evidences cannot answer the open questions on the reason behind the high accuracy, nor the error estimates of the dynamical properties, they are strong evidence on the applicability of AdResS beyond equilibrium simulations, and suggests a direction for theoretical investigations.

Currently the AdResS method has only been applied to model systems and small scale applications. The advantage in the efficiency is not very obvious in these studies. One important reason that prevents the AdResS method from large scale applications is the software implementation [22]. The software we used in the simulations is Gromacs 53. Currently the force computation kernel for the hybrid region is written in $\mathrm{C}$ language [54]. Comparing to the highly optimized assembly force computation kernels in Gromacs, the AdResS kernel is not competitive. Moreover, the current AdResS implementation keeps double resolution everywhere in the system[55]. Since in the coarse-grained region the force computation is very cheap, the majority of the computational cost is actually spent on keeping and integrating the atomistic DOFs. On straightforward solution is to remove the atomistic DOFs in the coarse-grained region, which was actually proposed by the very original paper of AdResS [1]. Owing to the fact that the coarse-grained interactions are usually "softer" than the atomistic interactions, one way of further boost the performance is to integrate the coarse-grained molecules with larger time-steps. If possible, the theoretical analyses on the consistency and the corresponding error estimates should be developed along the application of this adaptive time-step idea. All these technical difficulties would 
hopefully be solved in the near future.

[1] M. Praprotnik, L. Delle Site, and K. Kremer. Adaptive resolution molecular-dynamics simulation: Changing the degrees of freedom on the fly. The Journal of chemical physics, 123:224106, 2005.

[2] M. Praprotnik, L. Delle Site, and K. Kremer. Adaptive resolution scheme for efficient hybrid atomistic-mesoscale molecular dynamics simulations of dense liquids. Physical Review E, 73(6):066701, 2006.

[3] M. Praprotnik, S. Matysiak, L.D. Site, K. Kremer, and C. Clementi. Adaptive resolution simulation of liquid water. Journal of Physics: Condensed Matter, 19:292201, 2007.

[4] M. Praprotnik, L. Delle Site, and K. Kremer. Multiscale simulation of soft matter: From scale bridging to adaptive resolution. Annu. Rev. Phys. Chem., 59:545-571, 2008.

[5] A.B. Poma and L. Delle Site. Classical to path-integral adaptive resolution in molecular simulation: towards a smooth quantum-classical coupling. Physical review letters, 104(25):250201, 2010.

[6] S. Poblete, M. Praprotnik, K. Kremer, and L. Delle Site. Coupling different levels of resolution in molecular simulations. The Journal of chemical physics, 132:114101, 2010.

[7] M. Praprotnik, S. Poblete, and K. Kremer. Statistical physics problems in adaptive resolution computer simulations of complex fluids. Journal of Statistical Physics, pages 1-21, 2011.

[8] S. Fritsch, S. Poblete, C. Junghans, G. Ciccotti, L. Delle Site, and K. Kremer. Adaptive resolution molecular dynamics simulation through coupling to an internal particle reservoir. Physical Review Letters, 108:170602, 2012.

[9] S. Bevc, C. Junghans, K. Kremer, and M. Praprotnik. Adaptive resolution simulation of salt solutions. New J. Phys., 15:105007, 2013.

[10] Julija Zavadlav, Manuel Nuno Melo, Siewert J Marrink, and Matej Praprotnik. Adaptive resolution simulation of an atomistic protein in martini water. The Journal of chemical physics, 140(5):054114, 2014

[11] Julija Zavadlav, Manuel Nuno Melo, Ana Vicente Cunha, Alex H De Vries, Siewert J Marrink, and Matej Praprotnik. Adaptive resolution simulation of martini solvents. Journal of Chemical Theory and Computation, 10(6):2591-2598, 2014. 
[12] R. Delgado-Buscalioni, K. Kremer, and M. Praprotnik. Coupling atomistic and continuum hydrodynamics through a mesoscopic model: Application to liquid water. The Journal of Chemical Physics, 131:244107, 2009.

[13] B. Ensing, S.O. Nielsen, P.B. Moore, M.L. Klein, and M. Parrinello. Energy conservation in adaptive hybrid atomistic/coarse-grain molecular dynamics. Journal of Chemical Theory and Computation, 3(3):1100-1105, 2007.

[14] DM Heyes. Thermodynamic stability of soft-core lennard-jones fluids and their mixtures. The Journal of chemical physics, 132(6):064504, 2010.

[15] Q. Shi, S. Izvekov, and G.A. Voth. Mixed atomistic and coarse-grained molecular dynamics: simulation of a membrane-bound ion channel. The Journal of Physical Chemistry B, 110(31):15045-15048, 2006.

[16] Lin Shen and Hao Hu. Resolution-adapted all-atomic and coarse-grained model for biomolecular simulations. Journal of Chemical Theory and Computation, 10(6):2528-2536, 2014.

[17] S.O. Nielsen, P.B. Moore, and B. Ensing. Adaptive multiscale molecular dynamics of macromolecular fluids. Physical review letters, 105(23):237802, 2010.

[18] M. Praprotnik, S. Poblete, L. Delle Site, and K. Kremer. Comment on "adaptive multiscale molecular dynamics of macromolecular fluids". Physical Review Letters, 107(9):99801, 2011.

[19] Luigi Delle Site. Some fundamental problems for an energy-conserving adaptive-resolution molecular dynamics scheme. Physical Review E, 76(4):047701, 2007.

[20] Han Wang, Carsten Hartmann, Christof Schütte, and Luigi Delle Site. Grand-canonical-like molecular-dynamics simulations by using an adaptive-resolution technique. Physical Review $X, 3(1): 011018,2013$.

[21] Raffaello Potestio, Sebastian Fritsch, Pep Español, Rafael Delgado-Buscalioni, Kurt Kremer, Ralf Everaers, and Davide Donadio. Hamiltonian adaptive resolution simulation for molecular liquids. Physical review letters, 110(10):108301, 2013.

[22] Animesh Agarwal, Han Wang, Christof Schütte, and Luigi Delle Site. Chemical potential of liquids and mixtures via adaptive resolution simulation. The Journal of Chemical Physics, 141:034102, 2014.

[23] Luigi Delle Site. What is a multiscale problem in molecular dynamics? Entropy, 16(1):23-40, 2013.

[24] The long-range electrostatic interactions are also treated by the cut-off method, e.g. the 
Reaction-Field method.

[25] Han Wang, Christof Schütte, and Luigi Delle Site. Adaptive resolution simulation (AdResS): A smooth thermodynamic and structural transition from atomistic to coarse grained resolution and vice versa in a grand canonical fashion. Journal of Chemical Theory and Computation, 8(8):2878-2887, 2012.

[26] HJC Berendsen, JR Grigera, and TP Straatsma. The missing term in effective pair potentials. Journal of Physical Chemistry, 91(24):6269-6271, 1987.

[27] Han Wang, Christoph Junghans, and Kurt Kremer. Comparative atomistic and coarse-grained study of water: What do we lose by coarse-graining? The European Physical Journal E: Soft Matter and Biological Physics, 28(2):221-229, 2009.

[28] John D Weeks, David Chandler, and Hans C Andersen. Role of repulsive forces in determining the equilibrium structure of simple liquids. The Journal of chemical physics, 54(12):5237-5247, 1971.

[29] Han Wang, Christof Schütte, Giovanni Ciccotti, and Luigi Delle Site. Exploring the conformational dynamics of alanine dipeptide in solution subjected to an external electric field: A nonequilibrium molecular dynamics simulation. Journal of Chemical Theory and Computation, 10(4):1376-1386, 2014.

[30] Melville S Green. Markoff random processes and the statistical mechanics of time-dependent phenomena. ii. irreversible processes in fluids. J. Chem. Phys., 22:398-413, 1954.

[31] Ryogo Kubo. Statistical-mechanical theory of irreversible processes. I. General theory and simple applications to magnetic and conduction problems. J. Phys. Soc. Jpn., 12(6):570-586, 1957.

[32] William L Jorgensen, Jayaraman Chandrasekhar, Jeffry D Madura, Roger W Impey, and Michael L Klein. Comparison of simple potential functions for simulating liquid water. The Journal of chemical physics, 79(2):926-935, 1983.

[33] S. Pronk, S. Páll, R. Schulz, P. Larsson, P. Bjelkmar, R. Apostolov, M.R. Shirts, J.C. Smith, P.M. Kasson, D. van der Spoel, and Hess B. Lindahl E. Gromacs 4.5: a high-throughput and highly parallel open source molecular simulation toolkit. Bioinformatics, pages 1-10, 2013.

[34] Lars Onsager. Electric moments of molecules in liquids. Journal of the American Chemical Society, 58(8):1486-1493, 1936.

[35] I.G. Tironi, R. Sperb, P.E. Smith, and W.F. van Gunsteren. A generalized reaction field 
method for molecular dynamics simulations. The Journal of chemical physics, 102:5451, 1995.

[36] I. Fukuda, Y. Yonezawa, and H. Nakamura. Molecular dynamics scheme for precise estimation of electrostatic interaction via zero-dipole summation principle. The Journal of chemical physics, 134:164107, 2011.

[37] I. Fukuda. Zero-multipole summation method for efficiently estimating electrostatic interactions in molecular system. The Journal of Chemical Physics, 139:174107, 2013.

[38] J. Apostolakis, P. Ferrara, and A. Caflisch. Calculation of conformational transitions and barriers in solvated systems: Application to the alanine dipeptide in water. The Journal of chemical physics, 110(4):2099-2108, 1999.

[39] J.D. Chodera, N. Singhal, V.S. Pande, K.A. Dill, and W.C. Swope. Automatic discovery of metastable states for the construction of markov models of macromolecular conformational dynamics. The Journal of chemical physics, 126:155101, 2007.

[40] Jakub Kaminskỳ and Frank Jensen. Force field modeling of amino acid conformational energies. Journal of Chemical Theory and Computation, 3(5):1774-1788, 2007.

[41] D Gfeller, P De Los Rios, A Caflisch, and F Rao. Complex network analysis of free-energy landscapes. Proceedings of the National Academy of Sciences, 104(6):1817-1822, 2007.

[42] Nicolas Foloppe and Alexander D MacKerell Jr. All-atom empirical force field for nucleic acids: I. parameter optimization based on small molecule and condensed phase macromolecular target data. J. Comput. Chem., 21(2):86-104, 2000.

[43] Alexander D MacKerell, Michael Feig, and Charles L Brooks III. Extending the treatment of backbone energetics in protein force fields: Limitations of gas-phase quantum mechanics in reproducing protein conformational distributions in molecular dynamics simulations. $J$. Comput. Chem., 25(11):1400-1415, 2004.

[44] Jan-Hendrik Prinz, Bettina Keller, and Frank Noé. Probing molecular kinetics with markov models: metastable states, transition pathways and spectroscopic observables. Physical Chemistry Chemical Physics, 13(38):16912-16927, 2011.

[45] J.H. Prinz, H. Wu, M. Sarich, B. Keller, M. Senne, M. Held, J.D. Chodera, C. Schütte, and F. Noé. Markov models of molecular kinetics: Generation and validation. The Journal of chemical physics, 134:174105, 2011.

[46] C. Schütte, F. Noé, J. Lu, M. Sarich, and E. Vanden-Eijnden. Markov state models based on milestoning. The Journal of Chemical Physics, 134:204105, 2011. 
[47] Marco Sarich, Frank Noé, and Christof Schütte. On the approximation quality of markov state models. Multiscale Modeling \& Simulation, 8(4):1154-1177, 2010.

[48] Natasa Djurdjevac, Marco Sarich, and Christof Schütte. Estimating the eigenvalue error of markov state models. Multiscale Modeling \& Simulation, 10(1):61-81, 2012.

[49] F. Noé, C. Schütte, E. Vanden-Eijnden, L. Reich, and T.R. Weikl. Constructing the equilibrium ensemble of folding pathways from short off-equilibrium simulations. Proceedings of the National Academy of Sciences, 106(45):19011, 2009.

[50] Kai J Kohlhoff, Diwakar Shukla, Morgan Lawrenz, Gregory R Bowman, David E Konerding, Dan Belov, Russ B Altman, and Vijay S Pande. Cloud-based simulations on google exacycle reveal ligand modulation of gpcr activation pathways. Nature chemistry, 6(1):15-21, 2014.

[51] M. Senne, B. Trendelkamp-Schroer, A.S.J.S. Mey, C. Schütte, and F. Noé. Emma: A software package for markov model building and analysis. Journal of Chemical Theory and Computation, 8(7):2223-2238, 2012.

[52] The three-body correlation is the highest order correlation function that we could reach. The four-body correlation is out of the capability of numerical investigation, so it was not studied.

[53] Versions higher than 4.6 support AdResS.

[54] Brian W Kernighan, Dennis M Ritchie, and Per Ejeklint. The C programming language, volume 2. prentice-Hall Englewood Cliffs, 1988.

[55] In the coarse-grained region, the atomistic DOFs are not used in the force computations, but they are kept and updated in every numerical integration step. 